\title{
Water quality Prediction Model Based on fuzzy neural network
}

\author{
Fan Liao and Chunxia Zhao \\ Henan University of Chinese Medicine, Zhengzhou, China
}

\begin{abstract}
Keywords: water quality of aquaculture; dissolved oxygen prediction; principle component analysis; differential evolution algorithm; FNN
\end{abstract}

\begin{abstract}
This article proposes a dissolved oxygen prediction model for water quality about aquaculture to solve the problems like low accuracy and poor robustness of traditional prediction methods about water quality based on principal component analysis (PCA), fuzzy neural network(FNN), and differential evolution combined with BP algorithm (DEBP). This model can establish nonlinear dissolved oxygen prediction model of water quality about aquaculture though collecting principle components about indicators of aquatic ecological environment based on PCA, reducing the vector dimension as input in the model, using differential evolution algorithm to optimize the weighting parameters of FNN, and automatically acquiring optimal parameters. Based on this model, we conducted the prediction analysis about online water quality data of a shrimp culture pond in Zhanjiang from December 1 to December 12 in 2015, and the results of the trial indicates that: this model achieves a good predictive effect, compared with the BP-FNN model, the absolute error of $95.8 \%$ of tested samples of the PCA-FNN-DEBP model is less than $20 \%$ and the maximum error is $0.22 \mathrm{mg} / \mathrm{L}$, both of these two parameters are better than BP-FNN prediction model. The PCA-FNN-DEBP algorithm is not only fast and accurate, but also able to provide decision basis for adjustment and management of water quality in shrimp culture industry.
\end{abstract}

\section{Introduction}

Dissolved oxygen is a very important water quality indicator about fishery water, the condition of dissolved oxygen has great effect on water quality and the growth of cultured species. At present, the identification of dynamic changes of dissolved oxygen mostly adopts fixed-point and timing measurement and is mainly identified by managerial staff through observing the activity changes of living things bred in pond. This afterwards control usually has bad effect on the growth and development of cultured species. Therefore, before lack of oxygen, timely mastering the rules of dynamic changes of dissolved oxygen in the pond and making predictions about dissolved oxygen are the imperative problems needs to be solved in the process of aquaculture.

Fuzzy control does not need to build systematic accurate mathematic model, it can apply experts' knowledge, culturists' experience and other fuzzy linguistic information. However, fuzzy rules are decided by human's experience, its system has no self-learning ability and adaptive ability. Artificial neural networks are non-linear and have self-learning and adaptive abilities and other characteristics, but they are not able to show the reasoning function of human brains.FNN system has advantages of easily expressing human's knowledge and distributed information storage and learning ability based on combining fuzzy logic with neural networks, it is an effective tool to build and control complex system models. The prediction of dissolved oxygen in the culture pond is multivariable, non-linear with great time delay. FNN has good capability of non-linear approximation; therefore, applying FNN to making prediction about dissolved oxygen is a good solution. There are some researches about applying FNN to make predictions about aquaculture in foreign countries, and people in our country also use the method to make predictions about dissolved oxygen in the culture pond.

FNN training is mainly about the adjustment of fuzzy reasoning rules, and the adjustment of fuzzy reasoning rules is realized by adjusting weighting parameters of networks. Recently, some scholars use genetic algorithm $^{[5-6]}$, particle swarm optimization(PSO) algorithm ${ }^{[7]}$, ant colony 
algorithm $^{[8-9]}$, and other swarm intelligence algorithms to optimize the FNN parameters so as to improve the learning ability and prediction accuracy of algorithms.

Differential evolution algorithm (DE) is proposed by Stom R and others as a kind of random and parallel direct search algorithm based on genetic algorithm and other evolution algorithms. This algorithm can optimize the parameters and structures of neural-based fuzzy inference system, and amend miscellaneous membership function and the number of network nodes so as to optimize the fuzzy inference rules.

Dissolved oxygen prediction needs various kinds of environmental factors as input, but there exists some overlapped and tedious information among factors, if without screening of key factors, it will affect the result of dissolved oxygen prediction. Principle components analysis (PCA) can reduce the data dimension and eliminate the correlation of every independent variable while causing no damage or minimizing the loss of original indicator information.

For this reason, this article will combine PCA, DE algorithms with FNN, and build (PCA-FNN-DE) prediction model for dissolved oxygen of culture water quality based on DE optimization and fuzzy neural networks. After screening out the key impact factors related to dissolved oxygen with principle component analysis, and optimizing the parameters of fuzzy neural networks with differential evolution algorithm, the optimal parameter combination will be got to build the model of non-linear relations between ecological environment factors of shrimp culture, $\mathrm{pH}$ value, water temperature, ammonia nitrogen content and dissolved oxygen at some point in the future, besides, this article also will use the model to empirically analyze a shrimp culture pond in Zhanjiang for confirming the effectiveness of the algorithm.

\section{The Selection of Key Impact Factors about Dissolved Oxygen}

\section{A. Data Acquisition and Data Sources}

Take the water quality of one shrimp culture pond in Zhanjiang as the research object, use data acquisition system based on wireless sensor networks and timely obtain ecological environment data of shrimp culture. Adopt above wireless sensor network and take online samples of dissolved oxygen, $\mathrm{pH}$ value, turbidity, water temperature, ammonia nitrogen content, air pressure and other ecological environment data of shrimp culture every hour. Take 192 online data samples collected from December 1, 2014 to December 8, 2014 as research data source, choose 168 data samples of previous 7 days as training samples of FNN-DE, and 24 data samples of the last day as testing samples, and respectively train and validate the performance of prediction model of water quality.

The contribution rate of a principle component means the amount of information of original indicators reflected by this principle component. From Table 2, the first principle component can explain $48 \%$ of the amount of information of 5 original indicator variables, the second principle component can explain $24.5 \%$ of the amount of information of 5 original indicator variables, the third principle component can explain $13.7 \%$ of the amount of information of 5 original indicator variables 。 This shows that the first three principle components can explain $86.3 \%$ of the amount of information of 5 original variables, and these three principle components basically keep the information reflected by 5 original indicator variables. The three principle components conform to the rule of selecting principle components which need at least $85 \%$ of the accumulative contribution rate, therefore, choosing $\mathrm{pH}$ value, ammonia nitrogen value, water temperature as principle component indicators is basically the same as key impact factors about dissolved oxygen chosen by breeding experts with experience.

\section{B. The Establishment of Fuzzy Systematic Prediction Model of Dissolved Oxygen Based on Neural Networks}

\section{Modeling Method}

Build prediction model of dissolved oxygen based on PCA-FNN-DE by using the combination 
of principle component analysis, fuzzy neural networks and differential evolution algorithm. Firstly, make PCA analysis on the ecological environment data of shrimp culture with SPSS, screen out the key impact factors about dissolved oxygen as input vector of FNN prediction model, and optimize training of parameter combination of FNN prediction model with DE and BP algorithms to obtain the optimal prediction model and make predictions about dissolved oxygen at some point in the future. At the same time, predict through BP-FNN model which is only optimized by BP algorithm, and make comparative analysis on their prediction performance.

\section{FNN Model}

This article adopts improved fuzzy neural networks to build the prediction model of dissolved oxygen in the culture pond. The improved FNN is a network of 5 layers that respectively are input layer, fuzzy layer, inference layer, fuzzy normalized layer and output layer. As shown in Figure $1^{[14]}$, variables $\{\mathrm{x} 1, \mathrm{x} 2, \mathrm{x} 3\}$ in the input layer are corresponding to 3 principle components achieved after the principle component analysis, that are $\mathrm{pH}$ value of water in the culture pond, ammonia nitrogen value and water temperature. And the output layer is dissolved oxygen in the culture pond.

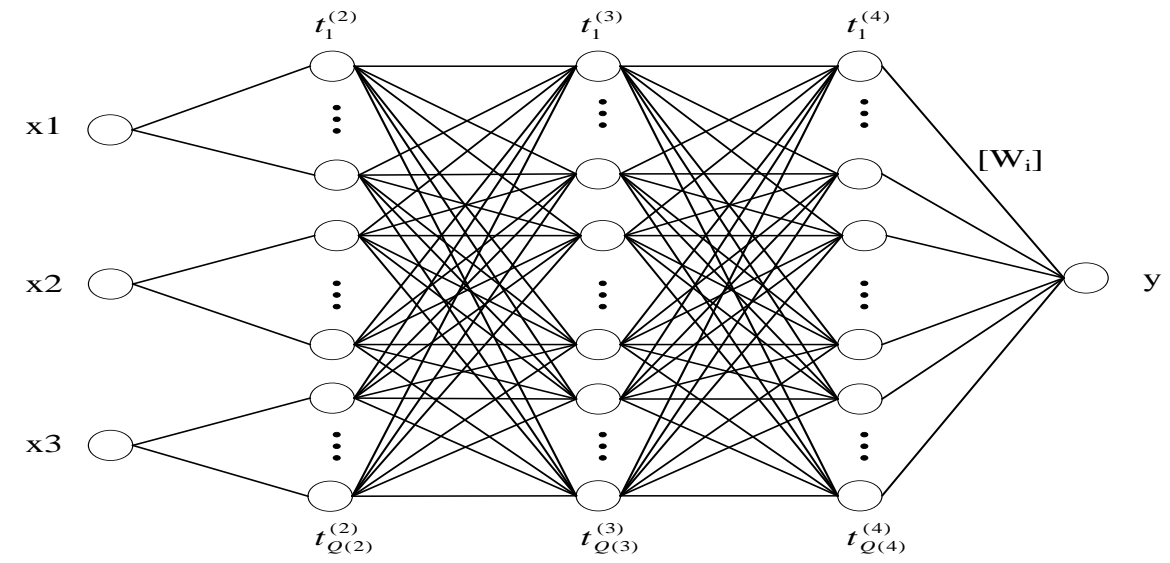

Figure 2 Fuzzy Neutral Network Model

Set the nodes number of input, output and the layer where it belongs of the j-th neuron unit of the k-th layer respectively as $S_{j}^{(k)}, t_{j}^{(k)}, U^{(k)}$.

The first layer is input layer, which shows the linguistic variables of input variables ( $\mathrm{pH}$ value, ammonia nitrogen value and water temperature) $x 1, x 2, x 3$, and the input layer has not weighting relations with the second layer and directly delivers the input to the second layer.

Node activation function is:

$$
t_{i}^{(1)}=S_{i}^{(1)}=X_{i}, \quad i=1,2, \cdots, u(1) 。
$$

The neuron number of the first layer is $U(1)=3$, with 3 corresponding principle components.

The second layer is fuzzy layer, composed of nodes of membership function with 21 neurons which show total fuzzy sets of input linguistic variables and complete the mapping from accurate input value to fuzzy value. Gaussian function has better smoothness than other membership functions, therefore choose it as membership function (5), and output membership values.

$$
h_{j}=\exp \left[-\frac{\left(x_{i}-c_{i j}\right)^{2}}{\mathrm{~b}_{j}^{2}}\right], \quad \mathrm{i}=1,2, \cdots, \mathrm{m}, \quad \mathrm{j}=1,2, \cdots, \mathrm{u}
$$

\section{Conclusion}

Dissolved oxygen is a key parameter in pond aquaculture environment which can precisely predict the dissolved oxygen in the pond and is very meaningful for aquaculture. This article proposes to predict the dissolved oxygen through FNN and practice the parameters of FNN based on EDBP, which can avoid FNN training entering the local minimum and improve the accuracy of prediction. Compared with traditional BP neural network, the model built in this article has higher accuracy and is able to meet the actual demand about dissolved oxygen adjustment in shrimp culture industry, besides, it provides reference data for management and prediction of water quality in other fields. 


\section{References}

[1] Stom R, Price K. Differential evolution - A simple and efficient adaptive scheme for global optimization over continuous spaces [R]. Berkeley: University of California, 2006.

[2] Liu bo, wang ling. Research progress on the differential evolution algorithm [J]. Control and decision, 2007, 22 (7) : 722.

[3] Jianhong Chen, liu Lang.zhi-yong zhou. Mining method based on principal component analysis and neural network optimization [J]. Journal of central south university: natural science edition, 2010, 9 (5) : 1967-1972.

[4] Zou Haiming Jiang Liangfu. Water quality evaluation method based on principal component analysis [J]. The practice and understanding of mathematics, 2008, 38 (8) : 85-90. 